\title{
THE CHANG-ŁOŚ-SUSZKO THEOREM IN A TOPOLOGICAL SETTING
}

\author{
PAUL BANKSTON
}

\begin{abstract}
The Chang-Łoś-Suszko theorem of first-order model theory characterizes universal-existential classes of models as just those elementary classes that are closed under unions of chains. This theorem can then be used to equate two model-theoretic closure conditions for elementary classes; namely unions of chains and existential substructures. In the present paper we prove a topological analogue and indicate some applications.
\end{abstract}

\section{INTRODUCTION AND THE MAIN THEOREM}

The Chang-Łoś-Suszko theorem of first-order logic states that an elementary class of relational structures is axiomatizable by a set of universal-existential sentences if and only if it is closed under unions of chains. There are some refinements of this famous result (e.g., the Keisler sandwich theorem, appearing in [8]); the one of most interest to us here appears as Theorem 1.2 in [18]. (We paraphrase slightly.)

Theorem 1.1. For any first-order theory $T$ and integer $k \geq 0$, the following three statements are equivalent:

(a) $T$ is $\Pi_{k+2}^{0}$ axiomatizable. (I.e., $T$ is axiomatizable via sentences in prenex normal form in which there are $k+2$ alternating blocks of quantifiers, the first consisting of universals.)

(b) The class of models of $T$ is closed under pre-images of embeddings of level $\geq k+1$. (I.e., if $A$ and $B$ are $L(T)$-structures and $B \models T$, then $A \models T$ also if there is an embedding $f: A \rightarrow B$ which satisfies the following: Given any $\Pi_{k+1}^{0}$ formula $\varphi\left(x_{1}, \ldots, x_{n}\right)$ and any $n$-tuple $\left\langle a_{1}, \ldots, a_{n}\right\rangle$ from $A$, then $A \models \varphi\left[a_{1}, \ldots, a_{n}\right]$ if and only if $\left.B \models \varphi\left[f\left(a_{1}\right), \ldots, f\left(a_{n}\right)\right].\right)$

(c) For any $\omega$-indexed direct system $A_{0} \stackrel{f_{0}}{\rightarrow} A_{1} \stackrel{f_{1}}{\rightarrow} \ldots$ of models of $T$, where each $f_{n}$ is an embedding of level $\geq k$, the limit is also a model of $T$.

In the present paper we explore an analogue of the equivalence of 1.1(b) and 1.1(c) above in the setting of compacta, the compact Hausdorff spaces. We also look at definability issues for certain well-known classes of compacta; in particular the class of continua, the connected compacta. Let us begin by stating the main theorem of the paper and briefly explaining what the words mean. In Section 2

2000 Mathematics Subject Classification. Primary 03C20, 54B35, 54C10, 54D30; Secondary 03C52, 06D05, 54D35, 54F15, 54F45, 54F55.

Key words and phrases. co-elementary hierarchy, co-existential mapping, ultracopower, ultracoproduct, compactum, continuum, covering dimension, multicoherence degree, Chang-Łoś-Suszko theorem. 
we give a proof that relies on several lemmas whose details are contained in the published literature, and in Sections 3, 4 and 5 we concentrate on applications.

Theorem 1.2. Let $\alpha$ be an ordinal number, and $\mathcal{K}$ a class of compacta.

(i) If $\mathcal{K}$ is closed under ultracopowers, images of co-elementary maps, and limits of $\omega$-indexed inverse systems with bonding maps of level $\geq \alpha$, then $\mathcal{K}$ is closed under images of maps of level $\geq \alpha+1$.

(ii) If $\mathcal{K}$ is closed under ultracoproducts and images of maps of level $\geq \alpha+1$, then $\mathcal{K}$ is also closed under limits of arbitrary inverse systems with bonding maps of level $\geq \alpha$.

For ease of language, let us define an inverse system of level $\geq \alpha$ to be an inverse system all of whose bonding maps are maps of level $\geq \alpha$. From Theorem 1.2 we may quickly infer the following graded topological reformulation of the ChangŁoś-Suszko theorem.

Corollary 1.3. Let $\alpha$ be an ordinal number, and $\mathcal{K}$ a co-elementary class of compacta. The following three statements are equivalent:

(a) $\mathcal{K}$ is closed under images of maps of level $\geq \alpha+1$.

(b) $\mathcal{K}$ is closed under limits of $\omega$-indexed inverse systems of level $\geq \alpha$.

(c) $\mathcal{K}$ is closed under limits of arbitrary inverse systems of level $\geq \alpha$.

Now for what the words mean. By a class of compacta, we understand a collection of compacta that is closed under homeomorphic copies. The principal construction used in our study is the ultracoproduct, an exact dualized version of the ultraproduct construction in model theory. Just as the ultraproduct of relational structures may be viewed as the limit of a directed system of products, so the ultracoproduct of compacta may be viewed as the limit of an inverse system of coproducts. In more detail, let $\left\langle X_{i}: i \in I\right\rangle$ be an indexed collection of compacta, with $\mathcal{D}$ an ultrafilter on $I$. For each $J \in \mathcal{D}$, we have the $J$-coproduct $X_{J}$, relative to the category of compacta and continuous maps, which is $\beta\left(\bigcup_{i \in J}\left(X_{i} \times\{i\}\right)\right)$, the Stone-Čech compactification of the disjoint union of the spaces $X_{i}$, for $i \in J$. And whenever $K \supseteq J \in \mathcal{D}$, there is the natural bonding map $f_{K J}: X_{J} \rightarrow X_{K}$ induced by inclusion. Now $\mathcal{D}$ is a directed set under reverse inclusion, and we take the ultracoproduct of the compacta $X_{i}$ with respect to the ultrafilter $\mathcal{D}$ to be the limit, denoted $\sum_{\mathcal{D}} X_{i}$, of this inverse system.

While the definition given above justifies our choice of terminology, there are other more useful ways to describe the ultracoproduct construction. One way (see, e.g., [2]) is to give $I$ the discrete topology and let $q: \bigcup_{i \in I}\left(X_{i} \times\{i\}\right) \rightarrow I$ be the obvious projection map. Applying the Stone-Cech functor $\beta()$, we view $\mathcal{D}$ as a member of $\beta(I)$; and it is not hard to show that $\sum_{\mathcal{D}} X_{i}$ is the pre-image of $\mathcal{D}$ under $\beta(q)$. This approach to the ultracoproduct was actually first used independently by J. Mioduszewski [14], in order to study $\beta([0, \infty))$. Of particular interest in this endeavor were the ultracoproducts of countably many copies of the closed unit interval. (See also the excellent survey [11] on this topic.)

The most flexible way to form $\sum_{\mathcal{D}} X_{i}$ for our present purposes is to take the following steps (see $[1,3])$ :

(i) Pick a lattice base $\mathcal{A}_{i}$ for $X_{i}, i \in I$ (i.e., $\mathcal{A}_{i}$ is a base for, as well as a sublattice of, the bounded lattice $F\left(X_{i}\right)$ of closed subsets of $\left.X_{i}\right)$;

(ii) form the ultraproduct lattice $\prod_{\mathcal{D}} \mathcal{A}_{i}$; and 
(iii) define the ultracoproduct to be the maximal spectrum $S\left(\prod_{\mathcal{D}} \mathcal{A}_{i}\right)$ (whose points are maximal filters in the ultraproduct lattice).

When each compactum $X_{i}$ is the same space $X$, we have the ultracopower of $X$ with respect to the ultrafilter $\mathcal{D}$, denoted $X I \backslash \mathcal{D}$. In addition to the projection $q: X \times I \rightarrow I$, there is now the projection $p: X \times I \rightarrow X$. And the restriction $p_{X, \mathcal{D}}$ of $\beta(p)$ to $X I \backslash \mathcal{D}$, called the codiagonal map, is a continuous mapping onto $X$. (Indeed, it is the image under the maximal spectrum functor of the canonical ultrapower embedding from $F(X)$ to $F(X)^{I} / \mathcal{D}$.) If $x \in X$ and $P \in X I \backslash \mathcal{D}$, then $x=p_{X, \mathcal{D}}(P)$ if and only if, for every open neighborhood $U$ of $x$, the ultrapower $U^{I} / \mathcal{D}$ includes a member of $P$.

We now turn our attention to the classification of maps between compacta. First we define a map $f: X \rightarrow Y$ to be co-elementary if there are ultrafilters $\mathcal{D}$ (on index set $I$ ) and $\mathcal{E}$ (on index set $J$ ) and a homeomorphism $h: X I \backslash \mathcal{D} \rightarrow Y J \backslash \mathcal{E}$ such that the function compositions $f \circ p_{X, \mathcal{D}}$ and $p_{Y, \mathcal{E}} \circ h$ are equal. (The KeislerShelah ultrapower theorem (see [8]) justifies our using this mapping criterion as the right (dualized) topological analogue of the notion of elementary embedding.) In parallel with the characterization of elementary classes in model theory in terms of closure under ultraproducts and elementary substructures, we define a class of compacta to be co-elementary if it is closed under ultracoproducts and images of co-elementary maps.

Next we define the co-elementary hierarchy of maps between compacta inductively as follows. Define $f: X \rightarrow Y$ to be a map of level $\geq 0$ if $f$ is a continuous surjection; for any ordinal $\alpha$, define $f: X \rightarrow Y$ to be a map of level $\geq \alpha+1$ if there is an ultrafilter $\mathcal{D}$ (on index set $I$ ) and a map $g: Y I \backslash \mathcal{D} \rightarrow X$, of level $\geq \alpha$, such that $f \circ g=p_{Y, \mathcal{D}}$. If $\lambda$ is a limit ordinal, we define $f$ to be of level $\geq \lambda$ if it is of level $\geq \alpha$ for all $\alpha<\lambda$.

Remark 1.4. $\quad$ (i) Maps of level $\geq n, n$ finite, are the topological counterparts of model-theoretic embeddings that are of level $\geq n$ in the sense of 1.1(b) above. Maps of level $\geq 1$, also called co-existential maps, correspond to existential embeddings between models. The reason for this (see, e.g., Theorem 1.1 in [18]) is that an embedding $f: A \rightarrow B$ between relational structures is of level $\geq n+1$ if and only if there is an ultrapower $A^{I} / \mathcal{D}$ and an embedding $g: B \rightarrow A^{I} / \mathcal{D}$, of level $\geq n$, such that $g \circ f$ is the canonical ultrapower embedding.

(ii) Co-elementary maps are of level $\geq \alpha$ for every ordinal $\alpha$; what is less obvious, is that maps of level $\geq \omega$ are already co-elementary. (While this is the way it should be, it is by no means trivial to prove, and is one of the main results (Theorem 2.10) in [4]).

(iii) Conspicuous in its absence from Theorem 1.2 and Corollary 1.3 is a syntactic component to correspond to 1.1(a). One possible way to remedy the situation would be to look at $S^{-1}[\mathcal{K}]$, the collection of all bounded lattices whose maximal spectra lie in $\mathcal{K}$. By arguments from [5] (see, esp., the proof of Theorem 6.1), $S^{-1}[\mathcal{K}]$ is an elementary class of lattices whenever $\mathcal{K}$ is a co-elementary class of compacta. In addition, if $\mathcal{K}$ satisfies one of the closure conditions, say $1.3(a)$, then $S^{-1}[\mathcal{K}]$ satisfies the dual condition for lattices. Because of Theorem 1.1, then, the following may be concluded from any of $1.3(a)-(c)$ : 
(d) $S^{-1}[\mathcal{K}]$ is an elementary class of bounded lattices, which is axiomatizable via a set of $\Pi_{\alpha+2}^{0}$ sentences.

But may we infer, say, 1.3(a) from (d)? If $X \in \mathcal{K}$ and $f: X \rightarrow Y$ is a map of level $\geq \alpha+1$ between compacta, does this imply that $Y$ is also in $\mathcal{K}$ ? The answer would be yes if it could be guaranteed that there is an embedding $g: B \rightarrow A$ of level $\geq \alpha+1$, where $S(B)=Y, S(A)=X$, and $S(g)=f$. But we do not know whether this is true in general. However, it is true that every continuous surjection $f: X \rightarrow Y$ between compacta is the image under $S()$ of the embedding $F(f): F(Y) \rightarrow F(X)$ between closed-set lattices, where $(F(f))(C):=f^{-1}[C]$ for any $C \in F(Y)$. So if $S^{-1}[\mathcal{K}]$ is $\Pi_{2}^{0}$ axiomatizable and $X_{0} \stackrel{f_{0}}{\leftarrow} X_{1} \stackrel{f_{1}}{\leftarrow} \ldots$ is an $\omega$-indexed inverse system of maps of level $\geq 0$ from $\mathcal{K}$, then $F\left(X_{0}\right) \stackrel{F\left(f_{0}\right)}{\longrightarrow} F\left(X_{1}\right) \stackrel{F\left(f_{1}\right)}{\longrightarrow} \ldots$ is an $\omega$-indexed direct system of embeddings (of level $\geq 0$ ) from $S^{-1}[\mathcal{K}]$, whose image under $S($ ) gives us the original system. Let $A$ be the limit of the direct system. Then, because $S^{-1}[\mathcal{K}]$ is a $\Pi_{2}^{0}$ class, we have $A \in S^{-1}[\mathcal{K}]$. And because the functor $S()$ converts direct limits to inverse limits, we know that $S(A)$, the limit of the original inverse system, is in $\mathcal{K}$.

By the last remark (1.4(iii)), we have the following topological reformulation of the original Chang-Łoś-Suszko theorem.

Corollary 1.5. Let $\mathcal{K}$ be a co-elementary class of compacta. The following four statements are equivalent:

(a) $\mathcal{K}$ is closed under images of co-existential maps.

(b) $\mathcal{K}$ is closed under limits of $\omega$-indexed inverse systems of level $\geq 0$.

(c) $\mathcal{K}$ is closed under limits of arbitrary inverse systems of level $\geq 0$.

(d) $S^{-1}[\mathcal{K}]$ is a $\Pi_{2}^{0}$-axiomatizable class of bounded lattices.

\section{PROOF OF THE MAIN THEOREM}

We first prove Theorem 1.2(i). Fix ordinal $\alpha$ and let $\mathcal{K}$ be a class of compacta that is closed under ultracopowers, images of co-elementary maps, and limits of $\omega$-indexed inverse systems of level $\geq \alpha$. Let $f_{0}: X_{0} \rightarrow Y_{0}$ be a map of level $\geq \alpha+1$, where $X_{0}$ is a compactum in $\mathcal{K}$. We need to show that $Y_{0}$ is also in $\mathcal{K}$. We have an ultracopower witness $g_{0}: Y_{0} I \backslash \mathcal{D} \rightarrow X_{0}$; so $p_{0}:=p_{Y_{0}, \mathcal{D}}=f_{0} \circ g_{0}$, and $g_{0}$ is a map of level $\geq \alpha$. Let $Y_{1}:=Y_{0} I \backslash \mathcal{D}$, and apply the functor ()$I \backslash \mathcal{D}$ iteratively to this mapping triangle. We let $X_{n+1}:=X_{n} I \backslash \mathcal{D}$, etc., so that we have an $\omega$-indexed inverse system $Y_{0} \stackrel{f_{0}}{\leftarrow} X_{0} \stackrel{g_{0}}{\leftarrow} Y_{1} \stackrel{f_{1}}{\leftarrow} X_{1} \stackrel{g_{1}}{\leftarrow} \ldots$, where $f_{n} \circ g_{n}=p_{n}$ for each $n<\omega$. Now by Proposition 2.3 and Corollary 2.4 in [4], each $p_{n}$ is co-elementary, and each $f_{n}$ (resp., each $g_{n}$ ) is a map of level $\geq \alpha+1$ (resp., level $\geq \alpha$ ). So the entire inverse system is of level $\geq \alpha$. Let $Z$ be the limit of this system. Because $\mathcal{K}$ is closed under ultracopowers, each $X_{n}$ is in $\mathcal{K}$. For each $n<\omega$, let $h_{n}:=g_{n} \circ f_{n+1}: X_{n+1} \rightarrow X_{n}$. By Proposition 2.5 in [4], each $h_{n}$ is a map of level $\geq \alpha$; so the $\omega$-indexed inverse sequence $X_{0} \stackrel{h_{0}}{\leftarrow} X_{1} \stackrel{h_{1}}{\leftarrow} \ldots$ is of level $\geq \alpha$ and comprises members of $\mathcal{K}$. Moreover, its limit is $Z$; hence $Z \in \mathcal{K}$. Now $Z$ is also the limit of the inverse system $Y_{0} \stackrel{p_{0}}{\leftarrow} Y_{1} \stackrel{p_{1}}{\leftarrow} \ldots$, a system with co-elementary bonding maps. At this point we cite a topological version of the elementary chains theorem of model theory, another main result in [4] (Theorem 3.2), to the effect that in such systems, the canonical projections from the limit to the factors are all co-elementary. Since $\mathcal{K}$ is closed 
under images of co-elementary maps, and $Z$ is in $\mathcal{K}$, we infer that $Y_{0}$ is in $\mathcal{K}$ as well. This completes the first half of the proof of Theorem 1.2.

To prove Theorem 1.2(ii), assume that $\mathcal{K}$ is now closed under ultracoproducts, as well as images of maps of level $\geq \alpha+1$. Let $\langle I, \leq\rangle$ be a directed set, with $\left\langle X_{i}, f_{i j}: i \leq j\right\rangle$ an inverse system of level $\geq \alpha$ from $\mathcal{K}$. (I.e., each $f_{i j}: X_{j} \rightarrow X_{i}$, $i \leq j$, is a map of level $\geq \alpha$, each $f_{i i}$ is the identity map on $X_{i}$, and, for $i \leq j \leq k$ in $I, f_{i k}=f_{i j} \circ f_{j k}$.) We may as well assume $I$ has no top element; otherwise there is nothing to prove. For each $i \in I$, let $[i, \infty)$ denote the ray $\{j \in I: i \leq j\}$. Then the collection of all rays satisfies the finite intersection property; hence there is an ultrafilter $\mathcal{D}$ on $I$ extending this collection. Letting $X$ be the limit of the inverse system above, we show that $X$ is in $\mathcal{K}$ by showing that $X$ is the image of a map of level $\geq \alpha+1$, whose domain is $\sum_{\mathcal{D}} X_{i}$ (which, by hypothesis, is in $\mathcal{K}$ ). For each $i \in I$, let $g_{i}: X \rightarrow X_{i}$ be the natural projection (defined by the equations $f_{j k} \circ g_{k}=g_{j}$ ). By Theorem 3.4 in [4] (a third main result of the paper, one whose argument may easily be extended to cover arbitrary inverse systems), each $g_{i}$ is a map of level $\geq \alpha$. By Corollary 2.4 in [4], then, so is the ultracoproduct map $\sum_{\mathcal{D}} g_{i}: X I \backslash \mathcal{D} \rightarrow \sum_{\mathcal{D}} X_{i}$. If we can produce a map $f: \sum_{\mathcal{D}} X_{i} \rightarrow X$ such that $f \circ \sum_{\mathcal{D}} g_{i}=p_{X, \mathcal{D}}$, then we will have demonstrated that this $f$ is a map of level $\geq \alpha+1$.

In order to obtain the required $f$, we first define maps $f_{j}: \sum_{\mathcal{D}} X_{i} \rightarrow X_{j}, j \in I$, in such a way that the equalities $f_{j k} \circ f_{k}=f_{j}$ hold whenever $j \leq k$ in $I$. This is easy. For each $j \in I$, define $F_{j}: \bigcup_{i \in[j, \infty)}\left(X_{i} \times\{i\}\right) \rightarrow X_{j}$ via the maps $f_{j i}: X_{i} \rightarrow X_{j}$. Since each ray $[j, \infty)$ is in $\mathcal{D}$, we may define $f_{j}$ to be the restriction of $\beta\left(F_{j}\right)$ to $\sum_{\mathcal{D}} X_{i}$. Continuity and the stated commutativity conditions are automatic.

We now can define $f: \sum_{\mathcal{D}} X_{i} \rightarrow X$ as the map uniquely specified by the equalities $g_{i} \circ f=f_{i}, i \in I$. We are done once we establish the equality $f \circ \sum_{\mathcal{D}} g_{i}=$ $p_{X, \mathcal{D}}$; and, by the special features of limits, this will be accomplished once we establish the equalities $f_{j} \circ \sum_{\mathcal{D}} g_{i}=g_{j} \circ p_{X, \mathcal{D}}$.

Assume the assertion is false and let $P \in X I \backslash \mathcal{D}$ witness the fact. We Set $Q:=\left(\sum_{\mathcal{D}} g_{i}\right)(P), x_{j}:=f_{j}(Q), y:=p_{X, \mathcal{D}}(P)$ and $y_{j}:=g_{j}(y)$. By assumption, $x_{j} \neq y_{j}$, so let $U$ and $V$ be disjoint open neighborhoods of $x_{j}$ and $y_{j}$ respectively. For each $i \in I$, set $U_{i}$ to be $f_{j i}^{-1}[U]$, if $i \in[j, \infty)$, and to be $X_{i}$ otherwise. On the one hand we have that $\prod_{\mathcal{D}} U_{i}$ includes a member of $Q$, so that $\left(\sum_{\mathcal{D}} g_{i}\right)^{-1}\left[\prod_{\mathcal{D}} U_{i}\right]$ includes a member of $P$. On the other hand we have that $g_{j}^{-1}[V]$ contains $y$; hence the ultrapower $\left(g_{j}^{-1}[V]\right)^{I} / \mathcal{D}$ also includes a member of $P$. Thus $\left\{i \in I: g_{i}^{-1}\left[U_{i}\right] \cap g_{j}^{-1}[V] \neq \emptyset\right\} \in \mathcal{D}$. But let $k \geq j$ in $I$. Then $g_{k}^{-1}\left[U_{k}\right] \cap g_{j}^{-1}[V]=g_{k}^{-1}\left[f_{j k}^{-1}[U]\right] \cap g_{j}^{-1}[V]=g_{j}^{-1}[U] \cap g_{j}^{-1}[V]=g_{j}^{-1}[U \cap V]=\emptyset$. Since $[j, \infty) \in \mathcal{D}$, we have a contradiction, and Theorem 1.2 is proved.

From the proof of Theorem 1.2(ii), we obtain the following.

Corollary 2.1. Let $\alpha$ be an ordinal, $\langle I, \leq\rangle$ a directed set, and $\left\langle X_{i}, f_{i j}\right\rangle$ an I-indexed inverse system of level $\geq \alpha$. If $\mathcal{D}$ is any one of a plethora of ultrafilters on $I$ that contain all the rays $[i, \infty), i \in I$, then the limit of this system is a level $\geq \alpha+1$ image of the ultracoproduct $\sum_{\mathcal{D}} X_{i}$. 


\section{APPLICATIONS TO DIMENSION}

In this section we consider some applications of Theorem 1.2 to the dimension theory of compacta. For any space $X$, the statement $" \operatorname{dim}(X) \leq n$," for $n<\omega$, means that every open cover $\mathcal{U}$ of $X$ refines to an open cover $\mathcal{V}$ of $X$ such that each point of $X$ lies in at most $n+1$ members of $\mathcal{V}$. The (Lebesgue) covering dimension $\operatorname{dim}(X)$ of $X$ is then the least $n<\omega$ for which that statement is true, if there is one, and $\infty$ otherwise.

A classic and easily-proved fact is that the class of compacta of covering dimension $\leq n$ is closed under limits of inverse systems of level $\geq 0$. This can also be proved, rather heavy-handedly, using Theorem 1.2; a better application, though, is the following new result.

Proposition 3.1. The covering dimension of the limit of an inverse system of level $\geq 1$ is the supremum of the covering dimensions of the compacta in the system.

Proof. By Theorem 2.6 in [5], the class of compacta of covering dimension $\leq n$ is closed under co-existential maps; by Theorem 2.2.2 in [1], it is closed under ultracopowers. A nearly identical argument shows the class to be closed under all ultracoproducts. By Remark 6.2(i) in [6], then, the class of compacta of covering dimension $n$ is closed under maps of level $\geq 2$. Now apply Theorem 1.2(ii), noting again that co-existential maps cannot raise covering dimension.

What makes Theorems 2.6 in [5] and 2.2.2 in [1] work is the theorem of E. Hemmingsen (Lemma 2.2, and its corollary, in [9]) to the effect that a normal Hausdorff space $X$ has covering dimension $\leq n$ if and only if, whenever $\left\{B_{1}, \ldots, B_{n+2}\right\}$ is a family of closed subsets of $X$, with $B_{1} \cap \cdots \cap B_{n+2}=\emptyset$, there exist closed subsets $\left\{F_{1}, \ldots, F_{n+2}\right\}$ such that:

(i) $B_{i} \subseteq F_{i}, 1 \leq i \leq n+2$,

(ii) $F_{1} \cup \cdots \cup F_{n+2}=X$, and

(iii) $F_{1} \cap \cdots \cap F_{n+2}=\emptyset$.

This is plainly a first-order lattice-theoretic statement, but its key feature is that it is independent of choice of lattice base for a compactum. To be more explicit, consider the lexicon $L_{B L}:=\langle\sqcup, \sqcap, \perp, \top\rangle$ of bounded lattices. Then we may view a lattice base $\mathcal{A}$ of a compactum $X$ is an $L_{B L}$-structure $\langle\mathcal{A}, \cup, \cap, \emptyset, X\rangle$. Now (see, e.g., [3] for details) an arbitrary $L_{B L}$-structure $A=\langle A, \sqcup, \sqcap, \perp, \top\rangle$ is isomorphic to a lattice base for some compactum if and only if $A$ satisfies:

(i) the axiom describing a bounded distributive lattice (a $\Pi_{1}^{0}$ sentence);

(ii) the "disjunctivity" axiom (a $\Pi_{2}^{0}$ sentence saying of every two distinct elements that there is a third element, not bottom, which is below one of the elements and disjoint from the other); and

(iii) the "normality" axiom (a $\Pi_{2}^{0}$ sentence saying of every two disjoint elements $a$ and $b$ that there are elements $a^{\prime}$ disjoint from $a$ and $b^{\prime}$ disjoint from $b$ such that the join of $a^{\prime}$ and $b^{\prime}$ is top).

$A$ is then called a normal disjunctive lattice, and we tacitly include these three axioms when we construct $L_{B L}$-sentences.

An $L_{B L}$-sentence $\varphi$ is base-free if, for any compactum $X$ and lattice base $\mathcal{A}$ for $X, \mathcal{A} \models \varphi$ if and only if $F(X) \models \varphi$. (An equivalent condition is: for any normal disjunctive lattice $A, A \models \varphi$ if and only if $F(S(A)) \models \varphi$.) If $\mathcal{K}$ is any class 
of compacta with the property that $S^{-1}[\mathcal{K}]$ is axiomatizable via a set of base-free sentences, then $\mathcal{K}$ is co-elementary.

The statement in Hemmingsen's theorem easily translates into a $\Pi_{2}^{0}$ sentence $\langle\operatorname{dim}\rangle_{\leq n}$ in the first-order language over $L_{B L}$, and this sentence has the quality we desire.

Proposition 3.2. $\langle\operatorname{dim}\rangle_{\leq n}$ is a base-free $\Pi_{2}^{0}$ sentence that defines the lattice bases of compacta of covering dimension $\leq n$.

Proof. Let $\mathcal{A}$ be a lattice base for the compactum $X$. The proof is an easy exercise, given Hemmingsen's characterization, once we note that if $B_{1}, \ldots, B_{k}$ are in $F(X)$, with $B_{1} \cap \cdots \cap B_{k}=\emptyset$, then it is possible to find $B_{1}^{\prime} \in \mathcal{A}$ such that $B_{1} \subseteq B_{1}^{\prime}$ and $B_{1}^{\prime} \cap\left(B_{2} \cap \cdots \cap B_{k}\right)=\emptyset$. Next, since $B_{2} \cap\left(B_{1}^{\prime} \cap B_{3} \cap \cdots \cap B_{k}\right)=\emptyset$, we may find $B_{2}^{\prime} \in \mathcal{A}$ such that $B_{2} \subseteq B_{2}^{\prime}$ and $B_{1}^{\prime} \cap B_{2}^{\prime} \cap B_{3} \cap \cdots \cap B_{k}=\emptyset$. Continue in this way to obtain the rest of the sets $B_{i}^{\prime}$.

A natural question to ask is whether the sentence $\langle\operatorname{dim}\rangle_{>n}$, the negation of $\langle\operatorname{dim}\rangle_{\leq n}$ relative to the $\left(\Pi_{2}^{0}\right)$ conditions for being a normal disjunctive lattice, clearly a base-free $\Pi_{3}^{0}$ sentence that says the covering dimension is $>n$, is (equivalent to) a base-free $\Pi_{2}^{0}$ sentence. The answer is no, but we need to establish some preliminary notions in order to show it. First of all, from Theorem 6.1 in [5], we know that if $\mathcal{K}$ is a co-elementary class of compacta that is closed under limits of inverse systems of level $\geq 0$, and if $X$ is an infinite member of $\mathcal{K}$, then $X$ is a continuous image of some $Y \in \mathcal{K}$ with the property that $Y$ has the same weight as $X$, and every continuous surjection from a member of $\mathcal{K}$ to $Y$ is co-existential. ( $Y$ is called coexistentially closed, relative to $\mathcal{K}$.) For example, we may choose $\mathcal{K}$ to be the class of all compacta; in which case there is a characterization of the "co-existentially closed compacta" (indulging in a slight abuse of language) as the zero-demensional compacta with no isolated points (Theorem 6.2 in [5]). Another important choice of $\mathcal{K}$ is the class of continua. By Theorem 4.5 of [4], every "co-existentially closed continuum" (also an abuse of language) is of covering dimension one. (More about co-existentially closed continua in Section 4.) With this information, we can now settle the question above.

Proposition 3.3. $\langle\operatorname{dim}\rangle_{>n}$ is a base-free $\Pi_{3}^{0}$ sentence, defining the lattice bases of compacta of covering dimension $>n$, having no $\Pi_{2}^{0}$ definition.

Proof. Clearly, for any compactum $X, F(X) \models\langle\operatorname{dim}\rangle_{>n}$ if and only if $\operatorname{dim}(X)>n$. It suffices to show that for fixed $n<\omega$, the class of compacta of dimension $>n$ is not closed under co-existential images. To see this, we consider the cases $n=0$ and $n>0$ separately.

In the case $n=0$, we let $X$ be any zero-dimensional compactum without isolated points, and take $f: X \times[0,1] \rightarrow X$ to be projection onto the first factor, where $[0,1]$ denotes the closed unit interval. Since $X$ is a co-existentially closed compactum, $f$ is a co-existential map from a compactum of positive dimension to one of zero dimension.

In the case $n>0$, we let $X$ be any co-existentially closed continuum, and take $f: X \times[0,1]^{n+1} \rightarrow X$, again, to be projection onto the first factor. Then $f$ is a co-existential map from a compactum of dimension $>n$ to one of dimension 1 .

Remark 3.4. $\quad$ (i) A compactum $X$ is a continuum just in case, whenever $A$ and $B$ are disjoint closed subsets of $X$ such that $A \cup B=X$, then either 
$A=\emptyset$ or $B=\emptyset$. The obvious translation of this textbook definition into a $\Pi_{2}^{0}$ sentence 〈cont〉 over $L_{B L}$ is easily shown to be base-free. This is more the exception than the rule; most textbook definitions of co-elementary classes do not translate so readily into base-free form. (See the definitions of indecomposable and of hereditarily indecomposable continua in Section 4.)

(ii) Often one can express a topological property in terms of first-order statements about closed-set lattices. While this may hold some interest, it does not guarantee that a co-elementary class is the outcome. As an exam-

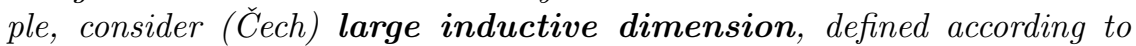
the scheme: Ind $(X)=-1$ if and only if $X$ is empty; and for fixed $n<\omega$, $\operatorname{Ind}(X) \leq n$ just in case, whenever $A$ and $B$ are disjoint closed subsets of $X$, there exists an open set $U$ containing $A$, with the closure $\bar{U}$ disjoint from $B$, such that $\operatorname{Ind}(\bar{U} \backslash U) \leq n-1$ (see [9]). It is not difficult to devise a first-order $L_{B L}$-sentence $\varphi_{n}$ with the property that for any compactum $X$ we have: $\operatorname{Ind}(X) \leq n$ if and only if $F(X) \models \varphi_{n}$. (We could start off by defining $\varphi_{0}$ to be $\langle\operatorname{dim}\rangle_{\leq 0}$.) But no matter how we may specify $\varphi_{n}$ for $n>0$, the sentence cannot be base-free. To show this, we use the construction, due to P. Vopěnka (see Proposition 18-10 of [17]), of compacta $X_{m}, 1 \leq m<\omega$, such that $\operatorname{dim}\left(X_{m}\right)=1$ and $\operatorname{Ind}\left(X_{m}\right)=m$. So now fix $m>n$. Then $F\left(X_{m}\right) \models \neg \varphi_{n}$. By the Löwenheim-Skolem theorem, we obtain a countable elementary sublattice $A_{m}$ of $F\left(X_{m}\right)$; thus $A_{m} \models \neg \varphi_{n}$, and hence (assuming $\varphi_{n}$ to be base-free) $F\left(S\left(A_{m}\right)\right) \models \neg \varphi_{n}$. But $S\left(A_{m}\right)$ is a metrizable compactum, so the major dimension functions agree for it. Thus $1=\operatorname{dim}\left(X_{m}\right)=\operatorname{dim}\left(S\left(A_{m}\right)\right)=\operatorname{Ind}\left(S\left(A_{m}\right)\right)$, and hence $F\left(S\left(A_{m}\right)\right) \models \varphi_{n}$.

\section{APPLICATIONS TO DECOMPOSABILITY IN CONTINUA}

A subcontinuum of a compactum is just a connected closed subspace; a continuum is decomposable if it is the union of two proper subcontinua, indecomposable otherwise. A continuum is hereditarily decomposable (resp., indecomposable) if every subcontinuum is decomposable (resp., indecomposable). It is these four properties that we consider in this section.

We begin the discussion with a result of R. Gurevič (Proposition 11 in [10]), that if $\left\langle X_{i}: i \in I\right\rangle$ is a family of compacta and $\mathcal{D}$ is an ultrafilter on $I$, then $\sum_{\mathcal{D}} X_{i}$ is a decomposable continuum if and only if $\left\{i: X_{i}\right.$ is a decomposable continuum $\} \in \mathcal{D}$. This tells us that both the classes $\{$ decomposable continua $\}$ and indecomposable continua\} are co-elementary, but it says nothing about the quantifier complexity of the firstorder descriptions of their respective classes of lattice bases.

Proposition 4.1. The class of indecomposable continua is closed under limits of inverse systems of level $\geq 0$. Thus $S^{-1}[\{$ indecomposable continua $\}]$ is $\Pi_{2}^{0}$ axiomatizable.

Proof. That this co-elementary class is closed under limits of inverse systems of level $\geq 0$ is well known; it also follows from the fact that, by Proposition 2.5 in [5], this class is closed under co-existential maps. Now apply Corollary 1.5.

Proposition 4.2. The class of decomposable continua is closed under limits if inverse systems of level $\geq 1$, but not under limits of inverse systems of level $\geq 0$. Thus $S^{-1}[\{$ decomposable continua $\}]$ is $\Pi_{3}^{0}$ axiomatizable, but not $\Pi_{2}^{0}$ axiomatizable. 
Proof. Since, from the last proof, indecomposability is preserved by maps of level $\geq 1$, it follows (see Theorem 2.5 in [6]) that decomposability is preserved by maps of level $\geq 2$. Thus, from the consequence (d) of Corollary 1.3 above, $S^{-1}$ [ decomposable continua $\left.\}\right]$ is $\Pi_{3}^{0}$ axiomatizable. On the other hand, decomposability is well known to fail to be preserved under limits of inverse systems of level $\geq 0$. (See, e.g., [16]). [One can also show decomposability fails to be preserved by co-existential maps: By Theorem 4.5 in [4], co-existentially closed continua are indecomposable. So let $X$ be one such; and form $Y$ by "spot-welding" two disjoint copies of $X$ at a single point, letting $f: Y \rightarrow X$ be the obvious projection map. Then $Y$ is decomposable, $X$ is indecomposable, and $f$ is co-existential.] Applying Corollary 1.5, we conclude that $S^{-1}[\{$ decomposable continua $\}]$ is not $\Pi_{2}^{0}$ axiomatizable.

It is easy to cook up an $L_{B L}$-sentence $\varphi$ that holds for $F(X)$ precisely when $X$ is a hereditarily decomposable continuum. However, $\varphi$ cannot be base-free, no matter how it is formulated.

Proposition 4.3. Let $\mathcal{D}$ be any nonprincipal ultrafilter on a countable set $I$. Then the $\mathcal{D}$-ultracopower of the closed unit interval is a continuum that is not hereditarily decomposable; hence the class of hereditarily decomposable continua is not co-elementary.

Proof. We lose no generality in letting $I$ be $\omega$. By Theorem 7.1 in [6], the class of hereditarily decomposable continua is closed under co-existential images. [Briefly, this is because:

(i) co-existential maps are weakly confluent (i.e., subcontinua in the range are images of subcontinua in the domain (see Theorem 6.2 in [6])); and

(ii) weakly confluent maps preserve hereditary decomposability (a nontrivial assertion, appearing as Exercise 13.66 in [16]).]

Let $f:[0,1] \rightarrow[0,1]$ take $t \leq 1 / 2$ to $2 t$, and $t \geq 1 / 2$ to $2-2 t$. $f$ is an indecomposable map; i.e., whenever $[0,1]$ is the union of two subcontinua, one of them is mapped by $f$ onto $[0,1]$. Then, by Theorem 2.7 in [16], the inverse limit $X$ of the sequence $[0,1] \stackrel{f}{\leftarrow}[0,1] \stackrel{f}{\leftarrow} \ldots$ (the "buckethandle continuum") is an indecomposable continuum. By Corollary 2.1 above, if $\mathcal{D}$ is any nonprincipal ultrafilter on $\omega$, then $X$ is a co-existential image of $[0,1] \omega \backslash \mathcal{D}$. Thus the ultracopower must contain an indecomposable subcontinuum.

Remark 4.4. $\quad$ (i) The ultracopower in Proposition 4.3 also fails to be locally connected. This gives an alternate proof of a result of $R$. Gurevič (Lemma 13 in [10]).

(ii) The statement in Proposition 4.3 that ultracopowers of the closed unit interval contain indecomposable subcontinua is known; both M. Smith [20] and J. -P. Zhu [21] independently gave constructions much more explicit than ours. Our proof of 4.3 is quite different from other approaches, though, and extends to a fairly broad class of continua. First, all you need is a continuum $X$ that admits an indecomposable self-map (e.g., any nondegenerate locally connected metrizable continuum). Then, by the argument in 4.3, any ultracopower $X \omega \backslash \mathcal{D}$, where $\mathcal{D}$ is a nonprincipal ultrafilter on $\omega$, will contain indecomposable subcontinua.

From Proposition 4.1, we know there is some base-free $\Pi_{2}^{0}$ sentence $\varphi$ such that for any compactum $X$ and lattice base $\mathcal{A}$ for $X, X$ is an indecomposable continuum 
if and only if $\mathcal{A} \models \varphi$. One can show without much difficulty that the standard definition does not translate into the $\varphi$ we want; what seems to be required is a new characterization of indecomposability.

Theorem 4.5. Let $X$ be a continuum, with $\mathcal{A}$ a lattice base for $X$. The following two statements are equivalent:

(a) $X$ is indecomposable.

(b) If $B \in \mathcal{A}$ and $X \backslash U \in \mathcal{A}$ are such that $\emptyset \neq U \subseteq B \neq X$, then there are $H, K \in \mathcal{A}$ with: $H \cap K=\emptyset, H \cup K=B$, and $H \cap U \neq \emptyset \neq K \cap U$.

Proof. Assume (a) holds, and let $B \in \mathcal{A}$ be proper, with $U$ a nonempty open set such that $X \backslash U \in \mathcal{A}$ and $U \subseteq B$. Fix $x \in U$, and let $C$ be the (connected) component of $B$ containing $x$. Since $C$ is a proper subcontinuum of $X$, and $X$ is indecomposable, we know (see Theorem 3-41 in [13]) that $C$ must have empty interior. Thus there is some point $y \in U \backslash C$. Since $x$ and $y$ are in different components of $B$, and $B$ is a compact Hausdorff space, a standard Zorn's lemma argument (see Theorem 2-14 in [13]) assures us that there is a separation $\{H, K\}$ of $B$ with $x \in H$ and $y \in K$. Thus we have $H \cap K=\emptyset, H \cup K=B$, and $H \cap U \neq \emptyset \neq K \cap U$. To complete the proof it suffices to show that $H$ and $K$ are in $\mathcal{A}$. Indeed, we know $H$ is an intersection $\bigcap_{i \in I} A_{i}$ of members of $\mathcal{A}$. Thus we know that $K=\bigcup_{i \in I}\left(B \backslash A_{i}\right)$. Since $K$ is also compact, we have $K=B \backslash\left(A_{i_{1}} \cap \cdots \cap A_{i_{n}}\right)$, for some finite subcollection of the sets $A_{i}$; hence $H=A_{i_{1}} \cap \cdots \cap A_{i_{n}} \in \mathcal{A}$. Likewise we have $K \in \mathcal{A}$ as well, and (b) is established.

Now assume (b) holds. Given $\emptyset \neq U \subseteq B \neq X$, with $U$ open and $B$ closed, it suffices to find a disconnection of $B$. Because $\mathcal{A}$ is a lattice base, it is possible to find sets $B^{\prime}$ and $U^{\prime}$ where: $B^{\prime}$ and $X \backslash U^{\prime}$ are in $\mathcal{A}, B^{\prime} \supseteq B, U^{\prime} \subseteq U, B^{\prime} \neq X$, and $U^{\prime} \neq \emptyset$. At this point we invoke (b) to find $H^{\prime}, K^{\prime} \in \mathcal{A}$, with $H^{\prime} \cap K^{\prime}=\emptyset$, $H^{\prime} \cup K^{\prime}=B^{\prime}$, and $H^{\prime} \cap U^{\prime} \neq \emptyset \neq K^{\prime} \cap U^{\prime}$. Set $H:=H^{\prime} \cap B$ and $K:=K^{\prime} \cap B$. Then $H$ and $K$ clearly form a disconnection of $B$, as long as we can show they are nonempty. But $\emptyset \neq H^{\prime} \cap U^{\prime}=H^{\prime} \cap B \cap U^{\prime}=H \cap U^{\prime} \subseteq H$; likewise for $K$. This completes the proof.

Let 〈indecomp $\rangle$ be the obvious translation of the condition 4.5(b) (along with the conditions for being a normal disjunctive lattice satisfying (cont $\rangle$ ). The following is immediate.

Corollary 4.6. 〈indecomp〉 is a base-free $\Pi_{2}^{0}$ sentence that defines the lattice bases of indecomposable continua.

Remark 4.7. Corollary 4.6 gives a new proof of the Gurevič result (Proposition 11 in [10]) that the classes $\{$ decomposable continua\} and \{indecomposable continua\} are co-elementary.

Denote by $\langle$ decomp $\rangle$ the complement of $\langle$ indecomp $\rangle$ relative to being a continuum. Then the following is immediate from Proposition 4.2.

Corollary 4.8. 〈decomp〉 is a base-free $\Pi_{3}^{0}$ sentence, defining the lattice bases of decomposable continua, having no $\Pi_{2}^{0}$ equivalent.

To summarize the situation, we know that:

(i) \{indecomposable continua $\}$ is $\Pi_{2}^{0}$ definable; 
(ii) $\{$ decomposable continua $\}$ is $\Pi_{3}^{0}$ definable, but not $\Pi_{2}^{0}$ definable; and

(iii) \{hereditarily decomposable continua $\}$ is not definable at all, in the sense meant here.

So what about the class \{hereditarily indecomposable continua\}? In [12] the authors use a 1977 theorem of J. Krasinkiewicz and P. Minc characterizing hereditary indecomposability in terms of closed sets only, and note that this characterization is base-free (see Theorem 1.2, and subsequent discussion, in [12]). This may be stated in a manner similar to Theorem 4.5 above as follows.

Theorem 4.9. Let $X$ be a continuum, with $\mathcal{A}$ a lattice base for $X$. The following two statements are equivalent:

(a) $X$ is hereditarily indecomposable.

(b) If $A, B \in \mathcal{A}$ are disjoint, $A \subseteq U$ and $B \subseteq V$, where $X \backslash U, X \backslash V \in \mathcal{A}$, then there are $H, K, M \in \mathcal{A}$ with: $A \subseteq H, B \subseteq M, H \cup K \cup M=X, H \cap M=\emptyset$, $H \cap K \subseteq V$, and $K \cap M \subseteq U$.

Let $\langle$ hered.indecomp $\rangle$ be the conjunction of "connected normal disjunctive lattice" with the "crookedness" condition 4.9(b). The following is immediate.

Corollary 4.10. 〈hered.indecomp〉 is a base-free $\Pi_{2}^{0}$ sentence that defines the lattice bases of hereditarily indecomposable continua. Thus the class of hereditarily indecomposable continua is co-elementary.

Remark 4.11. M. Smith [19] first proved that an ultracoproduct of hereditarily indecomposable continua is hereditarily indecomposable.

D. Bellamy proved in [7] that every metrizable continuum is a continuous image of a hereditarily indecomposable metrizable continuum. Armed with this, we were able to prove (Theorem 4.1 in [6]) that every co-existentially closed continuum is indecomposable, of covering dimension one; and, if metrizable, hereditarily indecomposable. We can now remove the metrizability condition. The following is proved in [12]; we offer another proof.

Theorem 4.12. Every continuum is a continuous image of a hereditarily indecomposable continuum of the same weight.

Proof. We begin by invoking a Löwenheim-Skolem-type theorem for compacta (see Theorem 3.1 in [5]), to the effect that: If $f: X \rightarrow Y$ is any continuous surjection between compacta, then there is a factorization $X \stackrel{g}{\rightarrow} Z \stackrel{h}{\rightarrow} Y$ of $f$, such that $g$ is co-elementary and $Z$ has any pre-assigned weight between those of $X$ and of $Y$.

So let $Y$ be any continuum. As in Remark 3.4(ii), we let $A$ be a countable elementary sublattice of $F(Y)$, and let $Y_{0}$ be $S(A)$, a metrizable continuum. Then there is a co-elementary map $c: Y \rightarrow Y_{0}$ induced by the inclusion $A \subseteq F(Y)$; so there are ultrafilters $\mathcal{D}$ (on index set $I$ ) and $\mathcal{E}$ (on index set $J$ ) and a homeomorphism $h: Y I \backslash \mathcal{D} \rightarrow Y_{0} J \backslash \mathcal{E}$ such that the function compositions $c \circ p_{Y, \mathcal{D}}$ and $p_{Y_{0}, \mathcal{E}} \circ h$ are equal. (The diagram commutativity here is nice, but unnecessary for this argument.) By Bellamy's theorem, let $g: Z_{0} \rightarrow Y_{0}$ be a continuous surjection, where $Z_{0}$ is a hereditarily indecomposable metrizable continuum. Then the ultracopower $Z_{0} J \backslash \mathcal{E}$ is a hereditarily indecomposable continuum by Corollary 4.10, and the composition $f:=p_{Y, \mathcal{D}} \circ h^{-1} \circ(g J \backslash \mathcal{E})$ is a continuous surjection from $X:=Z_{0} J \backslash \mathcal{E}$ to $Y$. Factoring this map as in the first paragraph above gives us 
what we want because co-elementary maps (indeed, co-existential maps) preserve hereditary indecomposability.

We now can remove the metrizability condition from Theorem 4.1 in [6].

Corollary 4.13. Every co-existentially closed continuum is a hereditarily indecomposable continuum of covering dimension one.

Proof. Suppose $Y$ is a co-existentially closed continuum. Then there is a continuous surjection $f: X \rightarrow Y$, where $X$ is a hereditarily indecomposable continuum. Then $f$ is co-existential; hence $Y$ is hereditarily indecomposable as well.

\section{APPLICATIONS TO MULTICOHERENCE DEGREE IN CONTINUA}

In this section we consider an application of Corollary 1.5 to the study of multicoherence degree in continua. This numerical measure of "connectedness" was invented by S. Eilenberg in the 1930s (see [16]) and is defined as follows. Given a continuum $X$, let $\mathcal{C}_{X}$ be the collection of pairs $\langle H, K\rangle$ of subcontinua of $X$, where $X=H \cup K$. If $\langle H, K\rangle \in \mathcal{C}_{X}$ and $H \cap K$ has a finite number $n \geq 1$ of components, we set (following tradition) $r(H, K):=n-1$; if the number of components is infinite, we set $r(H, K):=\infty$. The multicoherence degree $r(X)$ of $X$ is the maximum of the numbers $r(H, K),\langle H, K\rangle \in \mathcal{C}_{X}$, if such maximum exists, and is $\infty$ otherwise. So the multicoherence degree of an arc, a simple closed curve, and a figure-eight are, respectively, 0,1 , and 2 ; a continuum $X$ is called unicoherent just in case $r(X)=0$.

Our goal in this section is to show that multicoherence degree and covering dimension behave similarly within the present context. As a first step, we prove that the class of continua of any fixed finite multicoherence degree is co-elementary.

Theorem 5.1. Let $n<\omega$, with $\left\langle X_{i}: i \in I\right\rangle$ an indexed family of continua and $\mathcal{D}$ an ultrafilter on $I$. Then $r\left(\sum_{\mathcal{D}} X_{i}\right)=n$ if and only if $\left\{i \in I: r\left(X_{i}\right)=n\right\} \in \mathcal{D}$.

Proof. Clearly it suffices to show that, for $n<\omega, r\left(\sum_{\mathcal{D}} X_{i}\right) \geq n$ if and only if $\left\{i \in I: r\left(X_{i}\right) \geq n\right\} \in \mathcal{D}$. A further consequence of Theorem 3-41 in [13] (see the proof of 4.5) is that two disjoint subcompacta of a compactum, one of which is a component, may be separated by clopen sets. This, in turn, leads to the fact that a compactum $X$ has $\geq m$ components, $1 \leq m<\omega$, if and only if there is a partition of $X$ into $m$ nonempty subcompacta.

Suppose $\left\{i \in I: r\left(X_{i}\right) \geq n\right\} \in \mathcal{D}$. Then it is safe to assume that for each $i \in I$, we have $\left\langle H_{i}, K_{i}\right\rangle \in \mathcal{C}_{X_{i}}$ such that $r\left(H_{i}, K_{i}\right) \geq n$. Both $\sum_{\mathcal{D}} H_{i}$ and $\sum_{\mathcal{D}} K_{i}$ are subcontinua of $\sum_{\mathcal{D}} X_{i}$. Also, because the ultracoproduct operation on subsets commutes with finite unions and intersections, and because (see Proposition 1.5 in [1]) the Boolean lattice of clopen subsets of an ultracoproduct of compacta is the corresponding ultraproduct of the clopen set lattices of those compacta, we infer that $\left\langle\sum_{\mathcal{D}} H_{i}, \sum_{\mathcal{D}} K_{i}\right\rangle \in \mathcal{C}_{\Sigma_{\mathcal{D}} X_{i}}$ and $r\left(\sum_{\mathcal{D}} H_{i}, \sum_{\mathcal{D}} K_{i} \geq n\right.$. Consequently $r\left(\sum_{\mathcal{D}} X_{i}\right) \geq n$.

For the converse, suppose $r\left(\sum_{\mathcal{D}} X_{i}\right) \geq n$. Then there is some $\langle H, K\rangle \in \mathcal{C}_{\Sigma_{\mathcal{D}} X_{i}}$ with $r(H, K) \geq n$, and we may write $H \cap K=A_{0} \cup \cdots \cup A_{n}$, a union of pairwise disjoint nonempty subcompacta of $\sum_{\mathcal{D}} X_{i}$. Since we want to show $\left\{i \in I: r\left(X_{i}\right) \geq\right.$ $n\} \in \mathcal{D}$, there is nothing to prove in the case $n=0$.

So, assuming $n \geq 1$, we know that $H \cap K$ is disconnected; hence both $H \backslash K$ and $K \backslash H$ (being $\left(\sum_{\mathcal{D}} X_{i}\right) \backslash K$ and $\left(\sum_{\mathcal{D}} X_{i}\right) \backslash H$ respectively) are nonempty open 
sets in $\sum_{\mathcal{D}} X_{i}$. Given $\left\langle x_{i}: i \in I\right\rangle \in \prod_{i \in I} X_{i}$, there is just one point of $\sum_{\mathcal{D}} X_{i}$ containing $\prod_{\mathcal{D}}\left\{x_{i}\right\}$ as an element; call this point $\sum_{\mathcal{D}} x_{i}$. Then, by basic results in [1], the set of such points is dense in $\sum_{\mathcal{D}} X_{i}$. In light of this, we fix $\sum_{\mathcal{D}} x_{i} \in H \backslash K$ and $\sum_{\mathcal{D}} y_{i} \in K \backslash H$.

For each $k \leq n$, choose an open neighborhood $U_{k}$ of $A_{k}$ in such a way that the closures $\overline{U_{k}}$ are pairwise disjoint and miss both points $\sum_{\mathcal{D}} x_{i}$ and $\sum_{\mathcal{D}} y_{i}$. Let $R:=H \backslash\left(\bigcup_{k \leq n} U_{k}\right)$ and $S:=K \backslash\left(\bigcup_{k \leq n} U_{k}\right)$. Then $\sum_{\mathcal{D}} x_{i} \in R, \sum_{\mathcal{D}} y_{i} \in S$, and both $R$ and $S$ are subcompacta of $\sum_{\mathcal{D}} \bar{X}_{i}$. Moreover, $R$ and $S$ are disjoint because $R \cap S \subseteq(H \backslash K) \cap(K \backslash H)$.

For each $i \in I$, pick subcompacta $R_{i}, S_{i} \subseteq X_{i}$ such that $R \subseteq \sum_{\mathcal{D}} R_{i}, S \subseteq \sum_{\mathcal{D}} S_{i}$, and $\sum_{\mathcal{D}} R_{i} \cap \sum_{\mathcal{D}} S_{i}=\emptyset . R_{i}$ and $S_{i}$ may be chosen disjoint for each $i \in I$; so, in like fashion, we may choose pairwise disjoint subcompacta $A_{i 0}, \ldots, A_{i n} \subseteq X_{i}$ such that $\overline{U_{k}} \subseteq \sum_{\mathcal{D}} A_{i k}$ for $k \leq n$. Let $R_{i}^{*}:=R_{i} \cup\left(\bigcup_{k \leq n} A_{i k}\right)$ and $S_{i}^{*}:=S_{i} \cup\left(\bigcup_{k \leq n} A_{i k}\right)$. Then clearly $H \subseteq \sum_{\mathcal{D}} R_{i}^{*}$ and $K \subseteq \sum_{\mathcal{D}} S_{i}^{*}$. For each $i \in I$, let $C_{i}$ (resp., $D_{i}$ ) be the component of $R_{i}^{*}$ (resp., $S_{i}^{*}$ ) containing $x_{i}$ (resp., $y_{i}$ ). Because components may be separated from disjoint subcompacta via clopen sets, one can prove easily that "ultracoproducts of components are components of the ultracoproduct;" i.e., that $\sum_{\mathcal{D}} C_{i}$ (resp., $\sum_{\mathcal{D}} D_{i}$ ) is the component of $\sum_{\mathcal{D}} R_{i}^{*}$ (resp., $\sum_{\mathcal{D}} S_{i}^{*}$ ) containing $\sum_{\mathcal{D}} x_{i}$ (resp., $\left.\sum_{\mathcal{D}} y_{i}\right)$.

Thus we have $H \subseteq \sum_{\mathcal{D}} C_{i}$ and $K \subseteq \sum_{\mathcal{D}} D_{i}$; therefore $\bigcup_{k \leq n} A_{k}=H \cap K \subseteq$ $\sum_{\mathcal{D}} C_{i} \cap \sum_{\mathcal{D}} D_{i} \subseteq \sum_{\mathcal{D}} R_{i}^{*} \cap \sum_{\mathcal{D}} S_{i}^{*}=\bigcup_{k \leq n} \sum_{\mathcal{D}} A_{i k}$. For $i \in I, k \leq n$, let $B_{i k}:=C_{i} \cap D_{i} \cap A_{i k}$. Then $A_{k} \subseteq \sum_{\mathcal{D}} B_{i k}$, so each $\sum_{\mathcal{D}} B_{i k}$ is nonempty. Also $\bigcup_{k \leq n} \sum_{\mathcal{D}} B_{i k}=\sum_{\mathcal{D}} C_{i} \cap \sum_{\mathcal{D}} D_{i} \cap \bigcup_{k \leq n} \sum_{\mathcal{D}} A_{i k}=\sum_{\mathcal{D}} C_{i} \cap \sum_{\mathcal{D}} D_{i}$. From this it is immediate that $\left\{i \in I:\left\langle C_{i}, D_{i}\right\rangle \in \mathcal{C}_{X_{i}}\right.$ and $\left.r\left(C_{i}, D_{i}\right) \geq n\right\} \in \mathcal{D}$, therefore $\left\{i \in I: r\left(X_{i}\right) \geq n\right\} \in \mathcal{D}$. This completes the proof.

In order to use Theorem 5.1 to best advantage, we first state the following result of S. B. Nadler [15].

Theorem 5.2. Let $n\left\langle\omega\right.$, with $\langle I, \leq\rangle$ a directed set and $\left\langle X_{i}, f_{i j}\right\rangle$ an I-indexed inverse system consisting of continua of multicoherence degree $\leq n$ and surjective bonding maps. If $X$ is the limit of this system, then $r(X) \leq n$.

Remark 5.3. If, in Theorem 5.2, we assume $X$ to be locally connected, we obtain a quick proof as follows: By Corollary 2.1, there is an ultracopower $\sum_{\mathcal{D}} X_{i}$ and a co-existential map $f: \sum_{\mathcal{D}} X_{i} \rightarrow X$; by Theorem $5.1, r\left(\sum_{\mathcal{D}} X_{1}\right) \leq n$. Since $X$ is locally connected, $f$ is monotone (see Theorem 2.5 in [5]). Since $f^{-1}$ commutes with the finite Boolean operations on subsets and carries subcontinua to subcontinua, it is easy to see that $f$ cannot raise multicoherence degree.

Corollary 5.4. Let $f: X \rightarrow Y$ be a co-existential map between continua. Then $r(Y) \leq r(X)$.

Proof. This is immediate from Theorems 5.1 and 5.2, plus Corollary 1.5.

Corollary 5.5. Let $n\left\langle\omega\right.$, with $\langle I, \leq\rangle$ a directed set and $\left\langle X_{i}, f_{i j}\right\rangle$ an I-indexed inverse system consisting of continua of multicoherence degree $n$ and co-existential bonding maps. If $X$ is the limit of this system, then $r(X)=n$.

Proof. This is immediate from Theorem 5.2, Theorem 3.4 in [4] (all the projection maps from the limit are co-existential), and Corollary 5.4. 
Corollary 5.6. Let $f: X \rightarrow Y$ be a map of level $\geq 2$ between continua. Then $r(Y)=r(X)$.

Proof. This is immediate from Theorem 5.1, and Corollaries 5.5 and 1.3.

Remark 5.7. $\quad$ (i) The only previous significant result we are aware of in connection with Corollaries 5.4 and 5.6 is due to S. Eilenberg; namely (see, e.g., Theorem 13.33 in [16]) that quasi-monotone maps do not raise multicoherence degree.

(ii) Compare Corollary 5.5 with Theorem 2 in [15], which has the same conclusion, only with monotone surjective bonding maps. The two statements are entirely independent because co-existential maps need not be monotone (except when the range is locally connected), and vice versa.

(iii) By Theorems 5.1, 5.2 and Corollary 1.5, we know there must be, for each $n<\omega$, a base-free $\Pi_{2}^{0}$ sentence $\varphi_{n}$, in analogy with the dimension sentence $\langle\operatorname{dim}\rangle_{\leq n}$, expressing of a continuum that it has multicoherence degree $\leq$ $n$. We do not know of a formulation of such a sentence, however. (The negation of $\varphi_{n}$ relative to being a continuum, like the dimension sentence $\langle\operatorname{dim}\rangle_{>n}$, does not have a $\Pi_{2}^{0}$ expression because taking inverse limits can lower multicoherence degree by an arbitrary amount. Also co-existential maps can lower multicoherence degree. Indeed, as in the proof of Proposition 3.3, we can let $X$ be any co-existentially closed continuum, and let $f$ : $X \times C \rightarrow X$ be projection onto the first factor, where $C$ is the unit circle. Then $f$ is co-existential, $r(X)=0$, and $r(X \times C) \geq 1$.)

\section{REFERENCES}

[1] P. Bankston, Reduced coproducts of compact Hausdorff spaces, J. Symbolic Logic 52(1987), 404-424.

[2] Model-theoretic characterizations of arcs and simple closed curves, Proc. A. M. S. 104(1988), 898-904.

[3] _ Taxonomies of model-theoretically defined topological properties, J. Symbolic Logic 55 (1990), 589-603.

[4] _ A hierarchy of maps between compacta, J. Symbolic Logic 64 (1999), 1628-1644.

[5] Some applications of the ultrapower theorem to the theory of compacta, Applied Categorical Structures 8 (2000), 45-66.

[6] Continua and the co-elementary hierarchy of maps, Topology Proceedings 25 (2000), 45-62.

[7] D. Bellamy, Continuous mappings between continua, (Topology Conference, Greensboro, N. C., 1979), pages 101-111, Guilford College, Greensboro, N. C., 1980.

[8] C. C. Chang and H. J. Keisler, Model Theory third ed., North Holland, Amsterdam, 1990.

[9] R. Engelking, Outline of General Topology, North Holland, Amsterdam, 1968.

[10] R. Gurevič, On ultracoproducts of compact Hausdorff spaces, J. Symbolic Logic 53 (1988), 294-300.

[11] K. P. Hart, The Čech-Stone compactification of the Real line, in "Recent Progress in General Topology," M. Hušek, J. van Mill, eds., Elsevier Science Publishers, 1992, pages 318-351.

[12] K. P. Hart, J. van Mill, and R. Pol, Remarks on hereditarily indecomposable continua, Topology Proceedings 25 (2000), 179-206.

[13] J. G. Hocking and G. S. Young, Topology, Addison-Wesley, Reading, MA, 1961.

[14] J. Mioduszewski, On composants of $\beta \mathbf{R}-\mathbf{R}$, in Topology and Measure I, Part 2 (Zinnowitz, 1974), J. Flachsmeyer, Z. Frolík, and F. Terpe, eds., pages 257-283. Ernst-Moritz-ArndtUniversität zu Greifswald. 
[15] S. B. Nadler, Jr., Multicoherence techniques applied to inverse limits, Trans. A. M. S. 157 (1971), 227-234.

[16] _ Continuum Theory, An Introduction, Marcel Dekker, New York, 1992.

[17] K. Nagami, Dimension Theory, Academic Press, New York, 1970.

[18] H. Simmons, Existentially closed structures, J. Symbolic Logic, 37 (1972), 293-310.

[19] M. Smith, $\beta(X-\{x\})$ for $X$ not locally connected, Topology Appl. 26 (1987), 239-250.

$[20]-$ Layers of components of $\beta([0,1] \times \mathbf{N})$ are indecomposable, Proc. A. M. S. $114(1992)$, $1151-1156$.

[21] J. -P. Zhu, On indecomposable subcontinua of $\beta[0, \infty)-[0, \infty)$, in Proceedings of General Topology and Geometric Topology Symposium (Tsukuba, 1990), Topology Appl. 45 (1992), 261-274.

Department of Mathematics, Statistics and Computer Science, Marquette UniverSity, Milwaukee, WI 53201-1881

E-mail address: paulb@mscs.mu.edu 